\title{
Current status of HLA haplo-identical transplantation using post-transplantation cyclophosphamide
}

\author{
Hirohisa Nakamae \\ Hematology, Graduate School of Medicine, Osaka City University, Osaka, Japan
}

Outcomes of HLA haplo-identical transplantation using post-transplantation cyclophosphamide (PT/Cy-haplo) are similar to those of HLA-matched related/unrelated transplantation. Unlike classical HLA haplo-identical transplantation, PT/Cy-haplo does not require expensive T-cell depletion. Furthermore, accumulating evidences, including meta-analysis data, have indicated that PT/Cy-haplo may have a significant advantage over HLAmatched transplantation, concerning the low incidence of clinically significant, moderate-to-severe chronic graftversus-host disease. This clinical advantage of PT/Cy-haplo could contribute to economic medical benefits by reducing the cost and improve the quality of life of the patients. The establishment of PT/Cy-haplo could necessitate the recalibration of the donor selection algorithm.

(Journal of Hematopoietic Cell Transplantation 8(1): 16-21, 2019.)

\section{Introduction}

Haploidentical donors are being increasingly used as a usable donor source in the absence of HLA-matched related donors, especially with the prevailing global option of HLA haplo-identical transplantation using post-transplantation cyclophosphamide (PT/Cy-haplo). PT/Cy-haplo is being increasingly used in Japan as well, because of its acceptable safety profile. The use of cord blood for treating acute myeloid leukemia is decreasing in Europe. ${ }^{1}$

\section{What is the PT/Cy-haplo system?}

In PT/Cy-haplo, activated host-donor allo-reactive $\mathrm{T}$ cells proliferate in response to host-donor, dual allogeneic antigen stimulation. These cells can be selectively and efficiently eradicated by high dosage of $\mathrm{Cy}$ in a narrow time window, on days 3 or 4 after transplantation, because these $\mathrm{T}$ cells are sensitive to $\mathrm{Cy}$. Therefore, tolerance of dual alloreactive
T-cells can be induced by PT/Cy-haplo. ${ }^{2}$

In contrast, non-reactive T-cells that could be responsible for graft-versus-leukemia (GVL) effects, and protection against post-transplantation infection is spared because these T-cells are not sensitive to Cy. In PT/Cy-haplo, administration of tacrolimus and mycophenolate mofetil (MMF) is initiated after PT/Cy administration to avoid the blockage of Cy-induced tolerance. ${ }^{2}$

\section{Prospective clinical studies of PT/Cy-haplo}

Many prospective studies of PT/Cy-haplo have been reported, ${ }^{3-9}$ including a Japanese multi-center trial. These studies have demonstrated the safety and efficacy of PT/Cyhaplo, irrespective of the type of source 〔bone marrow (BM) or peripheral blood (PB)] (Table 1).

\footnotetext{
Submitted July 4, 2018; Accepted September 3, 2018; Published online, January 15, 2019. (Handling Editor: Tetsuya Nishida, Nagoya University Hospital)

Key words: HLA haplo-identical transplantation using post-transplantation cyclophosphamide (PT/Cy-haplo), mild acute graft-versus-host disease (GVHD), chronic GVHD, donor derived malignancy

Correspondence: Nakamae Hirohisa, Graduate School of Medicine, Osaka City University, 1-4-3 Asahi-machi, Abeno-ku, Osaka, 545-8585, Japan. E-mail: hirohisa@msic.med.osaka-cu.ac.jp dx.doi.org/10.7889/hct-18-008 (C) The Japan Society for Hematopoietic Cell Transplantation.
} 
Table 1. Prospective studies of HLA haplo-identical transplantation using post-transplantation cyclophosphamide

\begin{tabular}{lcclccc}
\hline Author & $\mathrm{n}$ & Type of graft & GVHD prophylaxis & II-IV aGVHD & cGVHD & Overall Survival \\
\hline Munchel et al. $^{3}$ & 210 & BM & CY50x2, Tac+MMF & $27 \%$ & $13 \%$ & $\begin{array}{l}60 \% \text { at } 1 \text { year } \\
48 \% \text { at } 2 \text { year } \\
41 \% \text { at } 3 \text { year }\end{array}$ \\
\hline Solomon et al. $^{4}$ & 20 & PBSC & CY50x2, Tac+MMF & $30 \%$ & $35 \%$ & $69 \%$ at 1 year \\
Solomon et al. $^{5}$ & 30 & PBSC & CY50x2, Tac+MMF & $43 \%$ & $56 \%$ & $78 \%$ at 2 year \\
Gaballa S et al. $^{6}$ & 60 & BM & CY50x2, Tac+MMF & $28 \%$ & $24 \%(2 \mathrm{ys})$ & $70 \%$ at 1 year \\
Brunstain et al. $^{7}$ & 50 & BM & CY50x2, Tac+MMF & $32 \%$ & $13 \%(1 \mathrm{ys})$ & $62 \%$ at 1 year \\
Sugita et al. $^{8}$ & 31 & PBSC & CY50x2, Tac+MMF & $23 \%$ & $15 \%(1 \mathrm{ys})$ & $45 \%$ at 1 year \\
Nakamae et al. $^{9}$ & 20 & PBSC & $\begin{array}{l}\text { CY25x2, CY } 25 \\
\text { Tac+MMF }\end{array}$ & $60 \%$ & $10 \%$ & $64 \%$ vs $44 \%$ at 1 year \\
\hline
\end{tabular}

BM, bone marrow; PBSC, peripheral blood stem cell; CY, clophosphamide; Tac, tacrolimus; MMF, mycophenolate mofetil.

\section{Comparison of outcomes after PT/Cy-haplo and HLA-matched related transplantation in a meta- analysis of case-control studies}

A recent meta-analysis compared PT/Cy-haplo and HLAmatched related transplants in nine case-control studies that included over 2,000 patients. ${ }^{10}$ In terms of acute graft-versushost disease (GVHD), non-relapse mortality, relapse, progression-free survival (PFS), and overall survival (OS), no differences between PT/Cy-haplo and HLA-matched transplant were evident. In addition, PT/Cy-haplo was significantly related to a lower occurrence of moderate-to-severe chronic GVHD. The meta-analysis findings suggested similar outcomes, except moderate-to-severe chronic GVHD, in case of PT/Cy-haplo and HLA-matched transplants. The findings indicate that PT/Cy-haplo might have an advantage over HLA-matched transplants, because the former is associated with a low incidence of moderate-to-severe chronic GVHD.

\section{Dilemma of classical HLA haplo-identical trans- plantation}

Non-selective T-cell depletion from the graft, through in vivo or ex vivo depletion, can delay immune reconstitution and increase immunosuppression. T-cell depletion is associated with increased risks of infection and relapse. On the contrary, weak in vivo or ex vivo depletion of T-cells results in a high incidence of GVHD; optimal T-cell modulation by in vivo or ex vivo depletion is very difficult. The difficulty in determining the optimal level of T-cell depletion could be a dilemma in classical HLA haplo-identical transplantation.

Theoretically, using PT/Cy can selectively eradicate alloreactive T-cells, which leads to a low incidence of GVHD and does not disrupt immune reconstitution. In addition, the high discontinuation rate of immune suppressants may contribute to a low transplantation-related mortality, which is synergistic with the low incidence of GVHD.

\section{Cellular mechanisms of T-cell reconstitution fol- lowing PT/Cy-haplo}

Based on the results of a clinical study conducted by an Italian group, the role of naive-derived $\mathrm{T}$ memory stem cells (TSCMs) is considered crucial in T-cell reconstitution following PT/Cy-haplo. ${ }^{11}$ Proliferating T cells are preferentially depleted by $\mathrm{Cy}$ on day 3 or 4 after transplantation. TSCMs, which are likely to be derived from naïve T-cells that were not eradicated by using PT/Cy, is the dominant peripheral T-cell subset one week after transplantation. In the subsequent weeks, naïve-derived TSCMs will produce memory cells by responding to exogenous antigens. Donor naïve T-cells specific for exogenous and self/tumor antigens remain in the host and largely contribute to immune reconstitution after PT/Cyhaplo.

\section{HLA-haploidentical blood or marrow transplanta- tion with high-dose post-transplant cyclophos- phamide at Johns Hopkins Hospital}

A total of 375 patients received PT/Cy-haplo, using a nonmyeloablative transplantation approach, at the Johns Hopkins Hospital (Baltimore, MD, USA) between 2004 and 2012. Recently, the results for 236 patients who underwent this treatment between 2004 and 2009 were reported. ${ }^{12}$ The cumulative incidence of acute GVHD was $28 \%$ for grades II-IV and $4 \%$ for grades III-IV, representing an extremely good control. 
Figure 1. Dose an excessive dose of PT/Cy partially overshadow graft-versus-leukemia (GVL) effects? Using an excessive dose of cyclophosphamide post transplantation may reduce the effects of GVL to some extent due to the robust elimination of alloreactive T-cells caused by the high dose of cyclophosphamide. The approach may result in the increase of the relapse rate.

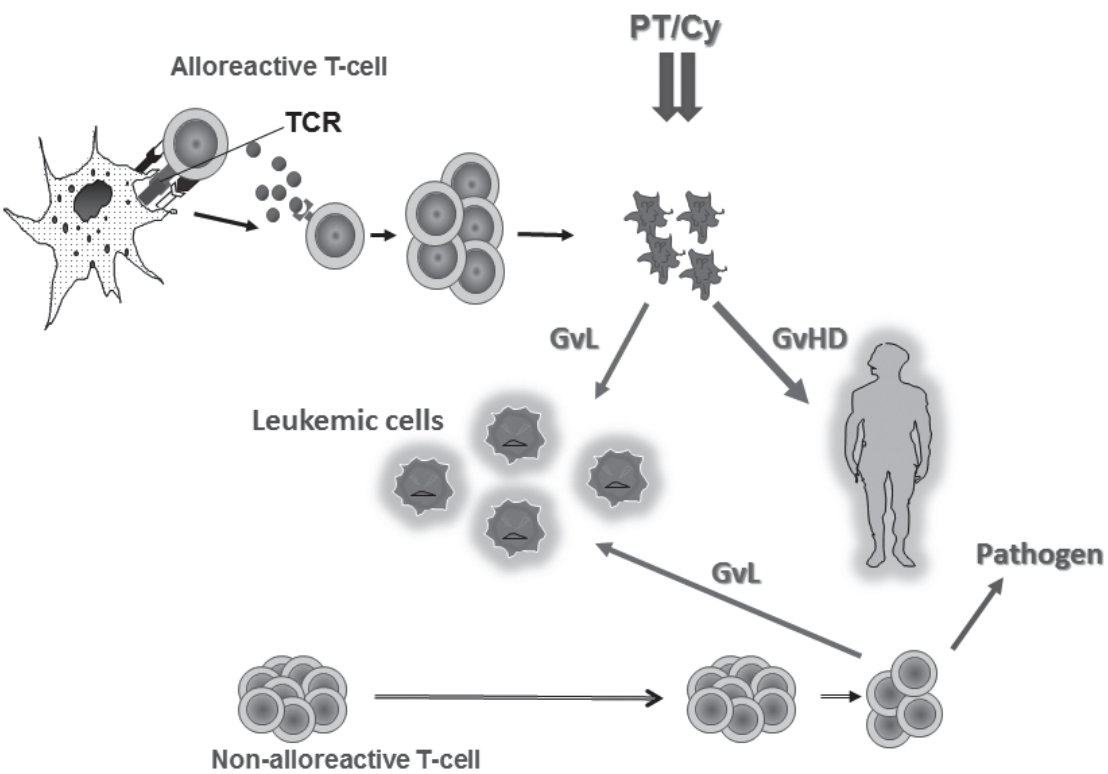

The cumulative incidence of non-relapse mortality was as low as $18 \%$. However, the relapse rate was $52 \%$, which is high, and is still a major issue to overcome. Use of bone marrow and/or weak conditioning may have been associated with the high relapse rate.

\section{Refined Disease risk index (DRI)-stratified out- comes in PT/Cy-haplo}

The refined DRI is an important indicator of the transplant outcome. A report comparing the outcomes between PT/Cyhaplo and HLA-matched bone marrow transplant from the group at Johns Hopkins showed comparable refined DRIstratified outcomes. ${ }^{13}$ In patients in the low, intermediate, and high/very-high risk groups, the 3-year event-free survival of PT/Cy-haplo and HLA-matched bone marrow transplantation were $65 \%$ vs. $66 \%, 39 \%$ vs. $31 \%$, and $25 \%$ vs. $15 \%$, respectively.

\section{Does an excessive dose of PT/Cy partially over- shadow graft-versus-leukemia (GVL) effects?}

Alloreactive T-cells may be at least partially relevant in GVL effects. We are considering the possibility that the GVL effects may be diminished to some extent by an excessive dose of Cy. Especially in cases of active disease, a strong eradication of alloreactive T-cells using high-doses of Cy may increase the relapse rate (Figure 1).

\section{Grade II acute GVHD improves PFS after PT/ Cy-haplo}

The high relapse rates noted with PT/Cy use may be associated with low rates of GVHD. The Johns Hopkins Hospital data demonstrated a significant relationship between mild grade II acute GVHD (also termed “good GVHD”) and a lower incidence of relapse and better probability of survival. ${ }^{14}$ These results also imply that GVHD and GVL cannot be clearly separated. Thus, we speculate that the suppressive effects of GVHD by PT/Cy use might, to some extent, contribute to the elimination of the GVL effects, as described above.

\section{Comparison of mobilized peripheral blood stem cells and unstimulated bone marrow as a graft source for PT/Cy-haplo}

A retrospective study evaluated the difference between the impact of outcomes of using PB and BM for PT/Cy-haplo. There were no significant differences in OS and non-relapse mortality. ${ }^{15}$ Incidences of grade II-IV acute and chronic GVHD were higher in case of PB. On the other hand, the risk of relapse was higher after BM transplant in patients with leukemia. Acute and chronic GVHD, which were more frequently observed after the transplantation of PB than of BM, might contribute to graft-versus-tumor effects. However, this analysis was adjusted for acute and chronic GVHD. Thus, the observed lower relapse risk in the PB group could not be sim- 
ply explained by the higher incidences of GVHD.

\section{Strategy for PT/Cy-haplo in our institute}

We hypothesized that PT/Cy use partially reduces GVL effects. Thus, to achieve substantial disease control, we have made unique modifications. These include intensified conditioning, use of peripheral stem cells as the only stem cell source, and a reduced dose of $\mathrm{PT} / \mathrm{Cy}{ }^{9}$ We are currently performing a prospective study using a total Cy dose of $75 \mathrm{mg}$ / $\mathrm{kg}$, with $50 \mathrm{mg} / \mathrm{kg}$ on day 3 and $25 \mathrm{mg} / \mathrm{kg}$ on day 4 (protocol: OCU16-2).

\section{Incidences of various infections following PT/Cy- haplo}

Although the incidences of infections including those due to bacteria and fungi in case of PT/Cy-haplo appeared to be higher than those in case of HLA-matched sibling transplantation, the incidences of infection in case of PT/Cy-haplo were comparable to those in case of HLA-matched and-mismatched unrelated transplantations, and unrelated cord blood transplantation. ${ }^{16}$

In terms of viral infections, the incidences of HHV6 reactivation at day 100 were significantly higher, but those of EBV reactivation at day 100 were significantly lower in case of PT/ Cy-haplo, than those in patients that received cells from matched or mismatched unrelated donors. ${ }^{17}$ The incidence of BK virus-associated cystitis varied from $11 \%$ to $75 \%$ in case of PT/Cy-haplo. ${ }^{4,18-20}$ Factors affecting the incidence of BK virus-associated cystitis included previous transplants and the occurrence of cytomegalovirus reactivation before cystitis. ${ }^{20}$ In addition, BK virus-associated cystitis obviously tended to occur at a high frequency in patients receiving high-dose busulfan-containing regimen.

\section{Impact of HLA disparity in haploidentical bone marrow transplantation followed by $\mathrm{PT} / \mathrm{Cy}$ administration}

Whether a different degree of HLA matching could affect outcomes in the PT/Cy-haplo setting is unknown. An Italian group analyzed 318 consecutive patients who received PT/ Cy-haplo with BM, and reported that the number of HLAmismatched antigens did not influence OS and non-relapse mortality. ${ }^{21}$ In addition, uni-vectorial graft-versus-host mis- matches were not related to the cumulative incidence of acute and chronic GVHD and relapse. Likewise, there was no significant relationship between the degree of HLA mismatch in the HVG direction and graft rejection.

\section{PT/Cy use after bone marrow transplantation is not associated with an increased risk of donor- derived malignancy}

Administering high-dose cyclophosphamide immediately after transplantation could theoretically cause the development of malignancy by disturbing the graft at a time of increased hematopoietic stress. ${ }^{22}$ Importantly, a competing risk analysis recently reported an estimated cumulative incidence of donor-derived malignancy of $1.4 \% .{ }^{17}$ The rate did not appear to be high when compared to other transplants, suggesting that using PT/Cy after bone marrow transplantation may not drastically increase the risk of donor-derived malignancy; however, further follow-ups will be required for the confirmation of this finding.

\section{Conclusions}

PT/Cy-haplo has several advantages, including readily available donors, lack of need for expensive or complicated T-cell depletion procedures, and demonstrated safety, mainly because of the low incidence of GVHD.

Recently, the use of PT/Cy-haplo has been increasing globally. If the safety profiles and feasibility of PT/Cy-haplo are confirmed prospectively, a paradigm shift in alternative donor selection could be necessary.

\section{Acknowledgments}

I am grateful to Professor Takanori Teshima, Department of Hematology, Faculty of Medicine, Hokkaido University, Professor Masayuki Hino and colleagues of Hematology, Graduate School of Medicine, Osaka City University, and members of Clinical Research Center of Hematology, Graduate School of Medicine, Osaka City University for supporting this work. This work was supported by the grant from the Japan Agency for Medical Research and Development (AMED, JP17ek0510012). 


\section{Conflict of interest disclosure}

H. $\mathrm{N}$ received Honoraria and Research funding from Chugai Pharmaceutical Co., Ltd., Bristol-Myers Squibb, Kyowa Hakko Kirin Co., Ltd., and Astellas Pharma, Inc.

\section{References}

1. Lee CJ, Savani BN, Mohty M, et al. Haploidentical hematopoietic cell transplantation for adult acute myeloid leukemia: a position statement from the Acute Leukemia Working Party of the European Society for Blood and Marrow Transplantation. Haematologica. 2017; 102: 1810-1822.

2. Luznik L, O’Donnell PV, Fuchs EJ. Post-transplantation cyclophosphamide for tolerance induction in HLA-haploidentical bone marrow transplantation. Semin Oncol. 2012; 39: 683693.

3. Munchel AT, Kasamon YL, Fuchs EJ. Treatment of hematological malignancies with nonmyeloablative, HLA-haploidentical bone marrow transplantation and high dose, post-transplantation cyclophosphamide. Best Pract Res Clin Haematol. 2011; 24: 359-368.

4. Solomon SR, Sizemore CA, Sanacore M, et al. Haploidentical transplantation using $\mathrm{T}$ cell replete peripheral blood stem cells and myeloablative conditioning in patients with high-risk hematologic malignancies who lack conventional donors is well tolerated and produces excellent relapse-free survival: results of a prospective phase II trial. Biol Blood Marrow Transplant. 2012; 18: 1859-1866.

5. Solomon SR, Sizemore CA, Sanacore M, et al. A. Total Body Irradiation-Based Myeloablative Haploidentical Stem Cell Transplantation Is a Safe and Effective Alternative to Unrelated Donor Transplantation in Patients Without Matched Sibling Donors. Biol Blood Marrow Transplant. 2015; 21: 12991307.

6. Gaballa S, Ge I, El Fakih R, et al. Results of a 2-arm, phase 2 clinical trial using post-transplantation cyclophosphamide for the prevention of graft-versus-host disease in haploidentical donor and mismatched unrelated donor hematopoietic stem cell transplantation. Cancer. 2016; 122: 3316-3326.

7. Brunstein CG, Fuchs EJ, Carter SL, et al; Blood and Marrow Transplant Clinical Trials Network. Alternative donor transplantation after reduced intensity conditioning: results of parallel phase 2 trials using partially HLA-mismatched related bone marrow or unrelated double umbilical cord blood grafts. Blood. 2011; 118: 282-288.

8. Sugita J, Kawashima N, Fujisaki T, et al; Japan Study Group for Cell Therapy and Transplantation. HLA-Haploidentical Peripheral Blood Stem Cell Transplantation with Post-Transplant Cyclophosphamide after Busulfan-Containing ReducedIntensity Conditioning. Biol Blood Marrow Transplant. 2015; 21: 1646-1652.
9. Nakamae H, Koh H, Katayama T, et al. HLA haploidentical peripheral blood stem cell transplantation using reduced dose of posttransplantation cyclophosphamide for poor-prognosis or refractory leukemia and myelodysplastic syndrome. Exp Hematol. 2015; 43: 921-929.

10. Gu Z, Wang L, Yuan L, et al. Similar outcomes after haploidentical transplantation with post-transplant cyclophosphamide versus HLA-matched transplantation: a meta-analysis of casecontrol studies. Oncotarget. 2017; 8: 63574-63586.

11. Roberto A, Castagna L, Zanon V, et al. Role of naive-derived T memory stem cells in T-cell reconstitution following allogeneic transplantation. Blood. 2015; 125: 2855-2864.

12. Fuchs EJ. HLA-haploidentical blood or marrow transplantation with high-dose, post-transplantation cyclophosphamide. Bone Marrow Transplant. 2015; 50 Suppl 2: S31-36.

13. McCurdy SR, Kanakry JA, Showel MM, et al. Risk-stratified outcomes of nonmyeloablative HLA-haploidentical BMT with high-dose posttransplantation cyclophosphamide. Blood. 2015; 125: 3024-3031.

14. McCurdy SR, Kanakry CG, Tsai HL, et al. Grade II Acute Graft-versus-Host Disease and Higher Nucleated Cell Graft Dose Improve Progression-Free Survival after HLA-Haploidentical Transplant with Post-Transplant Cyclophosphamide. Biol Blood Marrow Transplant. 2018; 24: 343-352.

15. Bashey A, Zhang MJ, McCurdy SR, et al. Mobilized Peripheral Blood Stem Cells Versus Unstimulated Bone Marrow As a Graft Source for T-Cell-Replete Haploidentical Donor Transplantation Using Post-Transplant Cyclophosphamide. J Clin Oncol. 2017; 35: 3002-3009.

16. Raiola AM, Dominietto A, di Grazia C, et al. Unmanipulated haploidentical transplants compared with other alternative donors and matched sibling grafts. Biol Blood Marrow Transplant. 2014; 20: 1573-1579.

17. Baker M, Wang H, Rowley SD, et al. Comparative Outcomes after Haploidentical or Unrelated Donor Bone Marrow or Blood Stem Cell Transplantation in Adult Patients with Hematological Malignancies. Biol Blood Marrow Transplant. 2016; 22: 2047-2055.

18. Raiola AM, Dominietto A, Ghiso A, et al. Unmanipulated haploidentical bone marrow transplantation and posttransplantation cyclophosphamide for hematologic malignancies after myeloablative conditioning. Biol Blood Marrow Transplant. 2013; 19: 117-122.

19. Castagna L, Crocchiolo R, Furst S, et al. Bone marrow compared with peripheral blood stem cells for haploidentical transplantation with a nonmyeloablative conditioning regimen and post-transplantation cyclophosphamide. Biol Blood Marrow Transplant. 2014; 20: 724-729.

20. Ruggeri A, Roth-Guepin G, Battipaglia G, et al. Incidence and risk factors for hemorrhagic cystitis in unmanipulated haploidentical transplant recipients. Transpl Infect Dis. 2015; 17: 822-830.

21. Raiola AM, Risitano A, Sacchi N, et al. Impact of HLA Dispar- 
ity in Haploidentical Bone Marrow Transplantation Followed by High-Dose Cyclophosphamide. Biol Blood Marrow Transplant. 2018; 24: 119-126.

22. Majzner RG, Mogri H, Varadhan R, et al. Post-Transplantation
Cyclophosphamide after Bone Marrow Transplantation Is Not Associated with an Increased Risk of Donor-Derived Malignancy. Biol Blood Marrow Transplant. 2017; 23: 612-617. 